\title{
Prevalence and Assessment of Knowledge and Practice Towards Hypertension among Bahir Dar City Communities, 2016: A Community Based Cross-sectional Study
}

Ayele Semachew Kasa* and Abdurehman Kelifa Shifa

School of Nursing, College of Medicine and Health Sciences, Bahir Dar University, Bahir Dar, Ethiopia

\begin{abstract}
Hypertension is a silent killer cardiovascular disease and is becoming a concerned public health challenges particularly in developing countries up to date. The global prevalence of raised blood pressure in adults aged 18 years and over was around $22 \%$ in 2014, and the number of people living with hypertension predicted as 1.56 billion at 2025 , increasing by $60 \%$. This study aimed to determine proportion of people with hypertension, and to assess knowledge and practice towards hypertension among Bahir Dar city Administration communities.
\end{abstract}

A community based cross-sectional study conducted on April, 2016 among Bahir Dar city administration communities with age greater than or equal to 20 years old. A multi-stage sampling technique used to select 388 study participants. The data were collected after oral informed consent secured for all study participants. Chi square test done to see whether there is an association between the predictor and outcome variable.

The prevalence rate of hypertension was $16.45 \%$. The percentage of knowledge and practice score for respondent's with poor score level was $71.8 \%$ and $84.3 \%$ respectively. Socio-demographic characteristics: education, occupation and health information concerning for hypertension associated with level of knowledge and practice toward hypertension.

Two hundred seventy-five $(71.8 \%)$ and $323(84.3 \%)$ of the respondents had poor knowledge and poor practice towards hypertension. As part of prevention programme, health education towards hypertension shall be planned and incorporated by Federal Ministry of Health along with other health topics provided by health extension workers and broadcasting Medias should focus towards cardiovascular and non-communicable diseases like hypertension.

Keywords: Hypertension; Cardiovascular disease; Knowledge

\section{Introduction}

Population growth and ageing, the number of people with uncontrolled HTN has risen over the years [1]. According to the seventh report of Joint National Committee prevention, detection, evaluation, and treatment of high blood pressure (JNC7) hypertension defined as a systolic blood pressure $\geq 140$ and a diastolic blood pressure $\geq 90$ based on the average of two or more accurate measurement taken during two or more contact with health care provider. But, it classified as pre-hypertension which is $120-139$ systolic and diastolic $80-89$, stage-I systolic of 140-159 and diastolic 90-99, stage-II systolic of $\geq 160$ and diastolic $\geq 100[2]$.

The global prevalence of raised blood pressure in adults aged 18 years and over was around 22\% in 2014 [1]. Prevalence of hypertension in Sub Saharan Africa, particularly in urban areas, was high $[3,4]$. The estimated prevalence rate of hypertension in overall Africa in adults aged 18 years and over $30 \%$ and $24.4 \%$ in Ethiopia [1]. The common risk factors for hypertension are obesity and weight gain, high sodium intake, low calcium and potassium intake, alcohol consumption, ageing, socioeconomic determinants psychological stress and low physical activity also heritability blood pressure is in the range of $15-35 \%[2,3,5]$.

Globally cardiovascular disease accounts for 17 million deaths a year, nearly one-third of the total, of these, complications of hypertension account for 9.4 million deaths worldwide. Every year hypertension is responsible for at least $45 \%$ of deaths due to heart disease, and $51 \%$ of deaths due to stroke [6]. By 2025 the projected number of people with hypertension expected to rise by $60 \%$ and reach 1.56 billion people. If left uncontrolled, hypertension causes stroke, myocardial infarction, cardiac failure, dementia, renal failure and blindness, causing human suffering and imposing severe financial and service burdens on health systems [1].
From different studies, prevalence of hypertension in urban is high due to low physical inactivity, better sedentary life, stress full environment. A study done in Addis Ababa showed that, the highest prevalence $30.2 \%$ [7] and a study done in South West Ethiopia showed the lowest prevalence $2.6 \%[8]$.

Now a day, prevalence of Non Communicable Disease including hypertension is increasing dramatically posing a double burden to countries of low socioeconomic status such as Ethiopia. Moreover because of weak health systems, the numbers of people with hypertension who undiagnosed, untreated and uncontrolled are also higher [6].

Measuring of knowledge and practices (KP) is a crucial element of hypertension control, but little is known about KP on hypertension from developing countries including ours, where hypertension has lately been recognized as a major health problem. Therefore, this study aimed to determine $\mathrm{KP}$ and prevalence on hypertension among general population of Bahir Dar city residents.

${ }^{*}$ Corresponding author: Dr. Ayele Semachew Kasa, School of Nursing, College of Medicine and Health Sciences, Bahir Dar University, Bahir Dar, Ethiopia, Tel: +251913959205; E-mail: ayeles@bdu.edu.et

Received August 29, 2017; Accepted September 14, 2017; Published Septembe 21, 2017

Citation: Semachew AK, Shifa AK (2017) Prevalence and Assessment of Knowledge and Practice Towards Hypertension among Bahir Dar City Communities, 2016: A Community Based Cross-sectional Study. J Hypertens (Los Angel) 6: 243. doi: 10.4172/2167-1095.1000243

Copyright: ( 2017 Semachew AK, et al. This is an open-access article distributed under the terms of the Creative Commons Attribution License, which permits unrestricted use, distribution, and reproduction in any medium, provided the original author and source are credited. 
Citation: Semachew AK, Shifa AK (2017) Prevalence and Assessment of Knowledge and Practice Towards Hypertension among Bahir Dar City Communities, 2016: A Community Based Cross-sectional Study. J Hypertens (Los Angel) 6: 243. doi: 10.4172/2167-1095.1000243

\section{Socio Demographic Characteristics}

The number of study participant enrolled in this study was 383 making the response rate $98.7 \%$, of these 205 (53.5\%) were male, 254 (66.3\%) married, 351 (91.6\%) Anhara ethnic group, 272 (71\%) orthodox religion follower, 134 (35\%) illiterate and 29(7.6\%), had Diploma, and $34(8.9 \%)$ had degree, $106(27.7 \%)$ merchant. The mean age of study participants were $38.24( \pm 17.2 \mathrm{SD})$.

\section{Information and knowledge about hypertension}

A total of 157 (41\%) had information about hypertension. Fifty six point one percent (56.1\%) of them didn't know the normal level of BP. Around 53\% of the sample size respond hypertension as high BP while about eight of them respond as high sugar. 33\% of the participants mentioned stress as a risk factor and 53\% answered that obesity is a risk factor for hypertension. Around twenty percent of the participant did not know complication of hypertension.

Regarding the diagnosis of hypertension, $70 \%$ of the participant answered that hypertension diagnosed through BP measurement while $1.6 \%$ of them thought it diagnosed by X-ray.

\section{Practice for prevention of hypertension}

From the total respondents 145 (37.8\%) of the respondents had previously measured their blood pressure. Regarding to physical exercise 115 (30\%) of the respondents reported that they do physical exercise regularly. From these 115 individuals $48.6 \%$ do physical exercise for less than 30 minutes per day and $8.8 \%$ perform for one hour per day.

Study respondents also asked about whether they addicted or not for different substances and $63(16.4 \%)$ addicted of these $30(7.8 \%)$ had alcohol addiction.

Overall among the total study participants 275 (71.8\%) and 59 (15.4\%) had poor and good knowledge for hypertension prevention and $323(84.3 \%)$ and $16(4.2 \%)$ had poor practice and good practice towards prevention of hypertension (Table 1).

\section{Prevalence of hypertension}

The overall prevalence of hypertension was $16.45 \%$. Prevalence of hypertension among female respondents was $8.6 \%$, for age groups between 20 and 40 years was $6.5 \%$ and $3.9 \%$ for age $41-60$ years. Prevalence of hypertension among illiterate respondents was $9.4 \%$ and prevalence of those who have degree and above educational status was $0.78 \%$. Among hypertensive cases, 39 (61.9\%) were newly diagnosed and the percentage of those reporting history of anti-hypertensive medication/hypertension were 19 (30.1\%), and participants who discontinued antihypertensive medication and whose BP was above normal (140/90 $\mathrm{mmHg}$ ) were 5 (7.94\%).

Among the total study participants 275 (71.8\%) and 59 (15.4\%) had poor and good knowledge for hypertension prevention respectively whereas $323(84.3 \%)$ and $16(4.2 \%)$ had poor practice and good practice towards prevention of hypertension respectively.

\begin{tabular}{|c|c|c|c|c|}
\hline & \multicolumn{2}{|c|}{ Knowledge } & \multicolumn{2}{c|}{ Practice } \\
\hline Score & $\mathbf{n}$ & $\mathbf{\%}$ & $\mathbf{n}$ & $\%$ \\
\hline Good & 59 & $15.4 \%$ & 16 & $4.2 \%$ \\
\hline Fair & 49 & $12.8 \%$ & 44 & $11.5 \%$ \\
\hline Poor & 275 & $71.8 \%$ & 323 & $84.3 \%$ \\
\hline Total & 383 & $100 \%$ & 383 & $100 \%$ \\
\hline
\end{tabular}

Table 1: Knowledge and practice level of study subjects regarding to hypertension, Bahir Dar city administration, 2016.
Hypertension was found prevalent (16.45\%), among hypertensive cases, 39 (61.9\%) were newly diagnosed and the number of people discontinuing medication was relatively increasing (7.94\%). The percentage of people with poor level of knowledge and practice was $71.8 \%$ and $84.3 \%$ respectively. Moreover, socio-demographic characteristics (educational status, occupation) and (marital status and age) has significant association with the level of knowledge and practice.

\section{Conclusion}

As part of prevention, health education programme to the level of specific knowledge for hypertension should plan and incorporated by policymaker along with other health topics provided by health extension workers and other health care providers to tackle incidence of hypertension. Furthermore, broadcasting medias should have a regular health education programme for hypertension.

\section{References}

1. World Health Organization (2014) Non-communicable disease profile. WHO, Geneva.

2. Suzanne C, Brende G, Janice L, Herry H (2010) Brunner Suddarths textbook of medical surgical nursing (12th edn.). Wolters Kluwer, India.

3. Giuseppe M, Robert F, Krzysztof N, Josep R, Alberto Z, et al. (2013) ESH E, $\mathrm{ESH} / \mathrm{ESC}$ guidelines for the management of arterial hypertension. European Heart Journal 34: 2159-2219.

4. Juliet A, Liam S, David AL (2007) Hypertension in Sub-Saharan Africa: A systematic review. J Am Heart Asso 50:1012-1018.

5. Dan LL, Anthony SF, Dennis LK, Stephen LH, Larry JJ, et al. (2012) Harrison principles of internal medicine (18th edn.). The McGraw-Hill Companies, USA.

6. World Health Organization (2013) A global brief on hypertension. WHO Geneva.

7. Tesfaye F, Byass P, Wall S (2009) Population based prevalence of high blood pressure among adults in Addis Ababa, uncovering a silent epidemic. BMC Cardiovascular Disease 9: 39.

8. Muluneh AT, Haileamlak A, Tessema F, Alemseged F, Woldemichael K, et al (2012) Population based survey of chronic non-communicable diseases at Gilgel Gibe Field Research Center, Southwest Ethiopia. Ethiop J Health Sci 22: 7-18. 